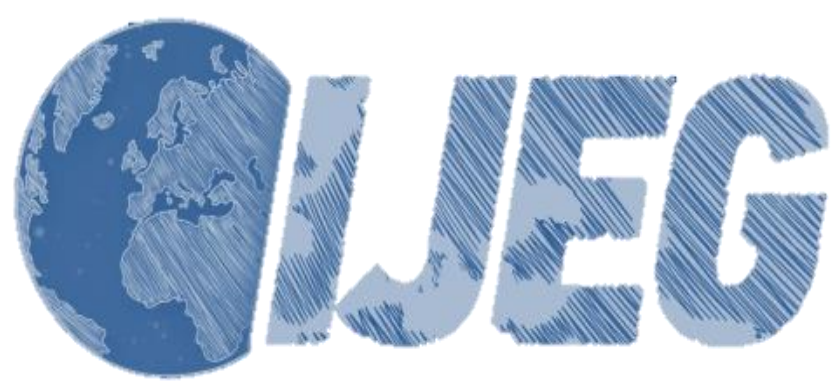

International Journal of Engineering and Geosciences (IJEG),

Vol; 3; , Issue; 1, pp. 012-019, February, 2018, ISSN 2548-0960, Turkey, DOI: 10.26833/ijeg.337806

\title{
COMPARISON OF GPS SATELLITE COORDINATES COMPUTED FROM BROADCAST AND IGS FINAL EPHEMERIDES
}

\author{
Tusat, E., ${ }^{1 *}$ Ozyuksel, F., ${ }^{2}$ \\ ${ }^{1}$ Selçuk University, Cumra School Of Applied Sciences, Department of Department of management Information \\ Systems, Cumra, Konya, Turkey (etusat@ selcuk.edu.tr); \\ $\stackrel{2}{2}$ Selcuk University, Engineering Faculty, Department of Geomatic Engineering, Konya, Turkey \\ (fethiozyuksel@gmail.com)
}

ORCID ID 0000-0003-4130-3764; ORCID ID 0000-0002-6868-8396

*Corresponding Author, Received: 12/09/2017, Accepted: 07/11/2017

\begin{abstract}
There are mainly two different orbital information, namely broadcast ephemerides and IGS final ephemerides (IGS rapid, ultra rapid, predicted and final ephemerides) used in the GPS positioning. The broadcast ephemerides used in practice and real time are obtained through assessments derived from the observations from the USA GPS reference stations. Broadcast ephemerides are formed (depending on GPS week) from satellite information and the accuracies they provide are adequate in many GPS applications. On the other hand, several parameters (for example, information about gravity area, improved satellite orbit information, etc.) need to be known in order to attain high accuracy in engineering and geodetic applications. Final ephemeris information can be downloaded from the related web sites via the internet. In this study, Keplerian motion and Keplerian orbital parameters will be explained briefly and extensive information about ephemerides and numerical applications will be given. Within this scope, for GPS satellites, ECEF coordinates of the satellites were computed using the broadcast ephemerides. The coordinates computed by using broadcast ephemerides were compared with the coordinates obtained from the IGS final orbits.
\end{abstract}

Keywords: GPS, Broadcast Ephemerides, IGS Ephemerides, Keplerian Orbital Parameters 


\section{INTRODUCTION}

Knowledge of ephemerides is an important issue for all GNSS applications because all ground positioning applications begin with the positions of GNSS satellites (Yoon, 2015). The computation and prediction of precise satellite orbits, together with appropriate observations and adjustment techniques is, for example, essential for the determination of;

-geocentric coordinates of observation stations,

-field parameters for the description of the terrestrial gravity field as well as for

the determination of a precise and high resolution geoid, -trajectories of land-, sea-, air-, and space-vehicles in realtime navigation,

-Earth's orientation parameters in space (Seeber, 1993)

Keplerian elements forming the fundamental information of the satellite orbit motion need to be known in order to make accurate orbit definitions in navigation and other relevant fields where satellite methods are used to determine positions.

Satellite motions are expressed through Kepler's Laws and are defined via six Keplerian orbital elements (Seeber 1993, Hoffmann-Wellenhof et al. 1994, Warren 2002). These are shown in Figure 1 and explained in Table 1.

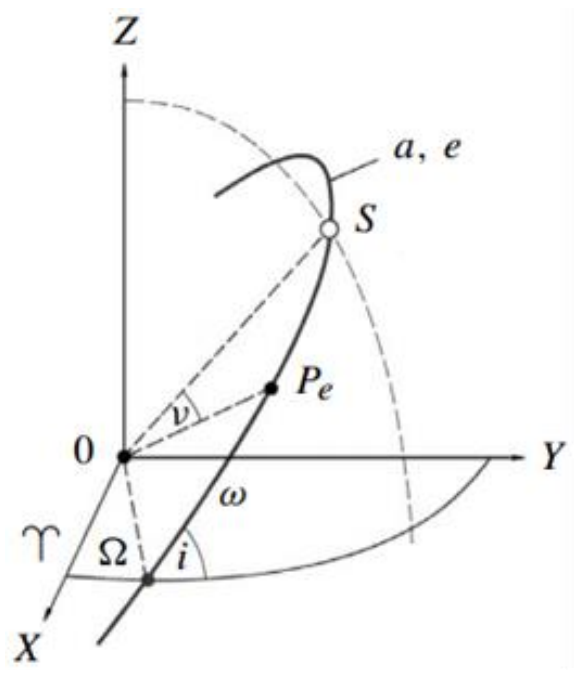

Figure 1 : Keplerian Orbital Elements (Seeber, 1993)

Three of the Keplerian orbital elements (a, e, P) describe the shape of the orbit while the other three elements (i, $\Omega$, $\omega$ ) enable orientation of the orbit in the ECEF (Earth Centered Earth Fixed) coordinate system.

Ephemerides data involving satellite orbital information are used to determine the position of a point on earth. Ephemerides data express Keplerian Orbital Information and data belonging to the momentary position of the satellite. Basis vector measured via GPS and point position accuracies vary depending on the accuracy of the ephemerides used in calculation.
Table 1 : Keplerian Orbital Elements (Warren, 2002)

\begin{tabular}{|c|l|}
\hline Parameter & \multicolumn{1}{|c|}{ Explanation } \\
\hline$\Omega$ & $\begin{array}{l}\text { Right ascension of ascending node } \\
\text { (measured as radian on the } \\
\text { equatorial plane) }\end{array}$ \\
\hline $\mathrm{i}$ & Inclination of the Orbital Plane \\
\hline$\omega$ & Argument of Perigee \\
\hline $\mathrm{a}$ & $\begin{array}{l}\text { Semi major axis of orbital plane } \\
\text { (meter) }\end{array}$ \\
\hline $\mathrm{e}$ & $\begin{array}{l}\text { Numerical eccentricity of ellipse; } \\
\text { e } \leq 0.01\end{array}$ \\
\hline $\mathrm{P}$ & Epoch of Perigee Passage \\
\hline
\end{tabular}

\section{GPS EPHEMERIDES}

Ephemerides in practical usage are broadcast ephemerides that constitute the control unit of GPS and are obtained through observations. Although broadcast ephemerides provide adequate accuracy in many applications, they may not be adequate for applications requiring high accuracy. Broadcast ephemerides are delivered to users as navigation messages (Seeber, 1993).

The error that occurs when the accuracy of the satellite position information broadcast in GPS Navigation message is low or when it is broadcast deliberately erroneously is called ephemeris error. This error is one of the disturbing effects that are hard to model. Therefore, it is important to take these disturbing effects, which are defined as ephemeris error, into consideration in computation of satellite orbits and this depends on proper measurement and modeling of forces affecting satellites (Tusat and Turgut, 2003). Since ephemeris error is a result of prediction of satellite positions, the size of this error will increase as one moves away from the reference epoch for ephemerides. When the matter is analyzed in terms of the user, the error that will be caused by satellite error in $\Delta \mathrm{r}$ size in fundamental components $(\Delta \mathrm{b})$ of base in b length can be expressed in the following equation (Kahveci and Yildiz, 2001).

$$
\frac{\Delta b(m)}{b(k m)}=\frac{\Delta r}{\rho_{R}^{S v}(k m)}
$$

Here, $\rho_{R}^{S v}$ denotes the satellite-receiver distance.

Thus, if satellite-receiver distance is taken to be approximately $20200 \mathrm{~km}$, errors in lengths that will be obtained for different ephemeris errors and base lengths on the basis of equation (1) are shown in Table 2 (Kahveci and Yildiz, 2001).

Table 2: Error Rates in Baseline from Ephemeris Errors

\begin{tabular}{|c|c|c|c|}
\hline $\begin{array}{c}\text { Ephemeris } \\
\text { Error }(\mathbf{m})\end{array}$ & $\begin{array}{c}\text { Baseline } \\
(\mathbf{k m})\end{array}$ & $\begin{array}{c}\text { Error } \\
(\mathbf{p p m})\end{array}$ & $\begin{array}{c}\text { Error } \\
(\mathbf{m m})\end{array}$ \\
\hline 2.5 & 1 & -- & -- \\
2.5 & 10 & 0.1 & 1 \\
2.5 & 100 & 0.1 & 12 \\
2.5 & 1000 & 0.1 & 124 \\
\hline 0.05 & 1 & -- & -- \\
0.05 & 10 & -- & -- \\
0.05 & 100 & 0.002 & 0.2 \\
0.05 & 1000 & 0.002 & 2.5 \\
\hline
\end{tabular}


The effect of ephemeris error is quite low for baselines of a few kilometers. However, the size of this error increases significantly in cases where baselines reach a few kilometers, which causes a problem in the use of GPS system in geodetic applications that require a high level of accuracy (Kahveci and Yildiz, 2001).

Main causes of the error in question involve atmospheric drag and pressure by solar radiation. Sizes of predicted orbit and real orbit may exhibit differences especially during periods of high solar activity. Accuracy of geocentric coordinates obtained through broadcast ephemerides is not better than $\pm 2-5 \mathrm{~cm}$ (Seeber, 1993).

\subsection{Broadcast Ephemerides}

Users need to know real time satellite positions and satellite system time in order to perform navigation tasks. Orbital information included in the data signal is broadcast via navigation message. Navigation message is determined by the Control Unit and transmitted to users by GPS satellites as "broadcast". The GPS navigation message file which contains the broadcast ephemeris gives the Keplerian parameters needed to compute the coordinates and clock correction for each satellite (Bidikar et al. 2014).

Broadcast ephemerides are used to compute the following items (Parkinson, 1996);

- satellite position at epoch,

- satellite velocity at epoch,

- three clock parameters per satellite,

- solar radiation pressure coefficients per satellite,

- y-axis acceleration bias,

- two clock parameters per monitor station, and

- one tropospheric scale factor per monitor station.

In the Kalman Filter process, predicted satellite positions are in the form of perturbation parameters and Keplerian elements (Figure 2). All parameters defining the satellite orbit and the state of the satellite clock are summarized in Table 3 below.

The parameters refer to a given reference epoch, toe for the ephemeris and toc for the clock, and they are based on a four hours curve fit (ICD, 1993). Hence, the representation of the satellite trajectory is achieved through a sequence of different disturbed Keplerian orbits (Seeber 1993).

The parameter sets in Table 3 are used to compute satellite time and satellite coordinates. The first group of the parameters are used for real satellite time. The second group defines a Kepler ellipse in reference epoch while the third group includes nine perturbation parameters.

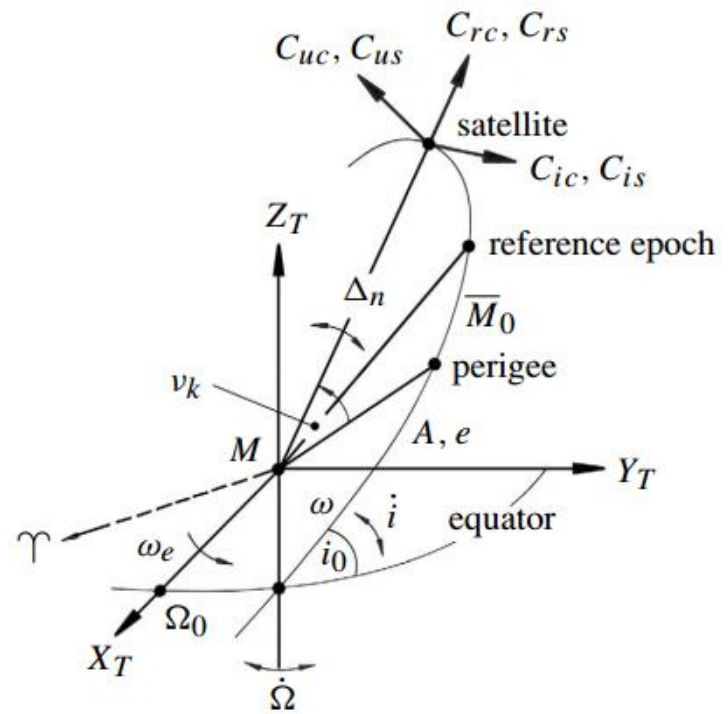

Figure 2: Keplerian and perturbed parameters in broadcast ephemerides (Seeber, 1993)

Table 3 : Parameters of Broadcast Ephemerides (Seeber, 1993)

\begin{tabular}{|c|c|}
\hline \multicolumn{2}{|r|}{ Time Parameters } \\
\hline$t_{0 e}$ & $\begin{array}{l}\text { Reference time, ephemerides } \\
\text { parameters [s] }\end{array}$ \\
\hline$t_{0 c}$ & Reference time, clock parameters [s] \\
\hline$a_{0}, a_{1}, a_{2}$ & $\begin{array}{l}\text { Polynomial coefficients for clock } \\
\text { corrections (bias [s], drift }[\mathrm{s} / \mathrm{s}] \text {, drift } \\
\text { rate }\left[\mathrm{s} / \mathrm{s}^{2}\right] \text { ) }\end{array}$ \\
\hline$I O D C$ & $\begin{array}{l}\text { Issue of Data, Clock, arbitrary } \\
\text { identification number }\end{array}$ \\
\hline \multicolumn{2}{|r|}{ Keplerian Parameters } \\
\hline $\begin{array}{l}\sqrt{\mathbf{a}}, \mathrm{e}, \mathrm{i}_{0} \\
\Omega_{0}, \omega, \mathrm{M}_{0}\end{array}$ & Keplerian elements of $\mathrm{T}_{0 \mathrm{e}}$ \\
\hline$I O D E$ & $\begin{array}{l}\text { Issue of Data, Ephemeris, arbitrary } \\
\text { identification number }\end{array}$ \\
\hline \multicolumn{2}{|r|}{ Perturbation Parameters } \\
\hline$\Delta n$ & $\begin{array}{l}\text { Mean motion difference from } \\
\text { computed value [semicircles/s] }\end{array}$ \\
\hline $\begin{array}{l}\text { di/dt (or } \\
\text { IDOT) }\end{array}$ & $\begin{array}{l}\text { Rate of change for inclination angle, } \\
\text { (radian/second) }\end{array}$ \\
\hline$\Omega$ & $\begin{array}{l}\text { Rate of change in ascending node } \\
\text { right ascension }\end{array}$ \\
\hline Cuc, Cus & $\begin{array}{l}\text { Correction coefficients for perigee } \\
\text { argument, (radian) }\end{array}$ \\
\hline $\mathrm{Crc}, \mathrm{Crs}$ & $\begin{array}{l}\text { Correction coefficients for geocentric } \\
\text { distance, (meter) }\end{array}$ \\
\hline Cic, $C i s$ & $\begin{array}{l}\text { Correction coefficients for } \\
\text { inclination angle, (radian) }\end{array}$ \\
\hline
\end{tabular}

\subsection{IGS Final Ephemerides}

Precise ephemerides and clock parameters depend on observations at monitor stations scattered across the world. Dual-frequency receivers that could measure both code phases and carrier phases of all visible satellites were established at some stations. Satellite errors can be purged of time errors of the station clock through use of 
Vol; 3; , Issue; 1, pp. 012-019, February, 2018,

high precision oscillators (rubidium-cesium atomic standard) (Seeber, 1993). Data files are in general compatible with SP3 (standard product 3) data format (Remondi, 1991; Hilla, 2002; IGS 2017).

Today, the most important source for final ephemerides and other GPS products is IGS. Production of IGS (International GNSS Service) orbital information began with an experimental GPS measurement campaign that took place on $21^{\text {st }}$ June 1992. (Kahveci and Yildiz, 2001) Unlike broadcast ephemerides, IGS orbits are formed from phase observations made in an intensive global network. Figure 3 shows IGS points in the world.

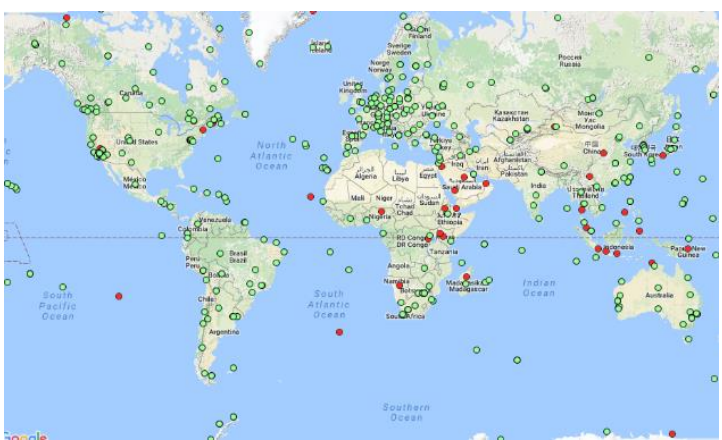

Figure 3 : IGS Network (IGS, 2017)

Today, IGS is responsible for collection, archiving and distribution of GPS measurements that could be used, with adequate accuracy, in scientific studies and engineering applications. These GPS measurements are used to obtain the following products (IGS, 2017).

- High accuracy GPS satellite ephemerides

- Earth rotation parameters (ERP)

- Coordinates of IGS monitor stations and their velocities

- Clock information belonging to GPS satellites and IGS monitor stations

- Computation of tropospheric zenith path delay

IGS products enable improvement and development of the ITRF system, determination of the movements of earth's crust, identification of changes on sea surface and provide high accuracy required by ionospheric studies. IGS performs these tasks within the following structure.

- A global observation network consisting of 506 stations

- Three global data centers

o CDDIS (Crustal Dynamics Data Information System at Goddard Space Flight Center, USA)

o IGN (Institut Geographique National, France)

o SIO (Scripps Institution Oceanography)

- Seven centers of analysis; CODE, NRCAN (EMR), ESA, JPL, GFZ, NGS and SIO.

The task of centers of analysis is to produce daily global data uninterruptedly (Kahveci and Yildiz, 2001). IGS produces four different pieces of orbital information according to orbits and clocks: IGS-Ultra-Speed, IGS Speedy, IGS Result orbital information (see Table 4).
Table 4 : IGS GPS satellite and clock accuracy (IGS, 2017)

\begin{tabular}{|c|c|c|c|c|c|}
\hline Type & \multicolumn{2}{|c|}{ Accuracy } & Latency & $\begin{array}{c}\text { Updat } \\
\text { es }\end{array}$ & $\begin{array}{c}\text { Sample } \\
\text { Interval }\end{array}$ \\
\hline \multirow[b]{2}{*}{ Broadcast } & orbits & $\sim 100 \mathrm{~cm}$ & \multirow[b]{2}{*}{ real time } & \multirow[b]{2}{*}{--} & \multirow[b]{2}{*}{ daily } \\
\hline & $\begin{array}{c}\text { Sat. } \\
\text { Clocks }\end{array}$ & $\begin{array}{c}\sim 5 \\
\text { ns } \\
\sim 2.5 \mathrm{RMS} \text { Sdev }\end{array}$ & & & \\
\hline \multirow{2}{*}{$\begin{array}{l}\text { Ultra-Rapid } \\
\text { (predicted } \\
\text { half) }\end{array}$} & orbits & $-5 \mathrm{~cm}$ & \multirow[b]{2}{*}{ real time } & \multirow{2}{*}{$\begin{array}{c}\text { at } 03, \\
09,15, \\
21 \\
\text { UTC }\end{array}$} & \multirow[b]{2}{*}{$15 \mathrm{~min}$} \\
\hline & $\begin{array}{l}\text { Sat. } \\
\text { Clocks }\end{array}$ & $\begin{array}{l}\sim 3 \mathrm{~ns} \quad \text { RMS } \\
\sim 1.5 \mathrm{~ns} \mathrm{Sdev}\end{array}$ & & & \\
\hline \multirow{2}{*}{$\begin{array}{l}\text { Ultra-Rapid } \\
\text { (observed } \\
\text { half) }\end{array}$} & orbits & $\sim 3 \mathrm{~cm}$ & \multirow[b]{2}{*}{$\begin{array}{l}3-9 \\
\text { hours }\end{array}$} & \multirow{2}{*}{$\begin{array}{c}\text { at } 03, \\
09,15, \\
21 \\
\text { UTC }\end{array}$} & \multirow[b]{2}{*}{$15 \mathrm{~min}$} \\
\hline & $\begin{array}{c}\text { Sat. } \\
\text { Clocks }\end{array}$ & $\begin{array}{c}\sim 150 \mathrm{ps} \\
\text { RMS } \\
\sim 50 \mathrm{ps} \mathrm{Sdev}\end{array}$ & & & \\
\hline \multirow[b]{2}{*}{ Rapid } & orbits & $\sim 2.5 \mathrm{~cm}$ & \multirow[b]{2}{*}{$\begin{array}{l}17-41 \\
\text { hours }\end{array}$} & \multirow{2}{*}{$\begin{array}{l}\text { at } 17 \\
\text { UTC } \\
\text { daily }\end{array}$} & $15 \mathrm{~min}$ \\
\hline & $\begin{array}{c}\text { Sat. \& } \\
\text { Stn. } \\
\text { Clocks }\end{array}$ & $\begin{array}{l}\sim 75 \mathrm{ps} \text { RMS } \\
\sim 25 \mathrm{ps} \text { Sdev }\end{array}$ & & & $5 \min$ \\
\hline \multirow[b]{2}{*}{ Final } & orbits & $\sim 2.5 \mathrm{~cm}$ & \multirow[b]{2}{*}{$\begin{array}{c}12-18 \\
\text { days }\end{array}$} & \multirow{2}{*}{$\begin{array}{l}\text { every } \\
\text { Thursd } \\
\text { ay }\end{array}$} & $15 \mathrm{~min}$ \\
\hline & $\begin{array}{c}\text { Sat. \& } \\
\text { Stn. } \\
\text { Clocks }\end{array}$ & $\begin{array}{l}\sim 75 \mathrm{ps} \text { RMS } \\
\sim 20 \mathrm{ps} \mathrm{Sdev}\end{array}$ & & & $\begin{array}{c}\text { Sat.: } 30 \mathrm{~s} \\
\text { Stn.: } 5 \\
\text { min }\end{array}$ \\
\hline
\end{tabular}

\section{MATERIAL AND METHOD}

So far, GPS satellite orbits have been explained. In this regard, GPS satellite coordinates were computed in the ECEF coordinate system using the IGS final (igs18632.sp3) and broadcast ephemerides (brdc2650.15n) data belonging to September 22, 2015 at 12.00 hours in order to investigate the effect of ephemerides information on coordinates, and comparisons were made. In the numerical application conducted, the effect of broadcast ephemerides belonging to the points on satellite coordinates was investigated by changing only the orbital information from among the selected parameters in the comparison.

\section{Computation of ECEF Coordinates from Satellite Orbits}

RINEX (Recevier INdependent EXchange format) navigation file data are used to compute a GPS satellite orbit or its position at a certain moment in ECEF coordinate system. (Figure 4).

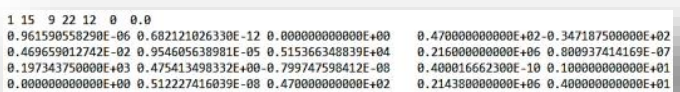

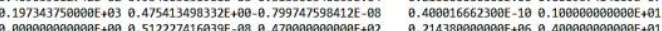

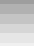

Figure 4 : RINEX data block for SV 1

Ephemerides parameters used in the computations are given Table 3. It is possible to compute highly accurate satellite coordinates in the ECEF coordinate system using these data in a certain algorithm. 


\section{Satellite Position Computation Algorithm}

Computation of the position of a satellite in ECEF coordinate system is quite simple. The algorithm to be used to this end is given in Table 5. $\boldsymbol{E}_{\boldsymbol{k}}$ and $\boldsymbol{V}_{\boldsymbol{k}}$ variables, which were not in linear correlation, were found using Newton-Raphson iteration technique.

Table 5 : Satellite Position Computation Algorithm

\begin{tabular}{|c|c|}
\hline$G M_{e}=3.986008 \times 10^{4} \mathrm{~m}^{3} / \mathrm{s}^{2}$ & $\begin{array}{l}\text { Gravitational } \\
\text { Constant }\end{array}$ \\
\hline$\dot{\Omega} e=7.292115167 \times 10^{-5} \mathrm{rad} / \mathrm{s}$ & Earth rotation rate \\
\hline$a=(\sqrt{a})^{2}$ & Semi-major axis \\
\hline$n_{0}=\sqrt{\frac{G M}{a^{3}}}$ & $\begin{array}{l}\text { Computed mean } \\
\text { motion }\end{array}$ \\
\hline$n=n_{0}+\Delta n$ & $\begin{array}{l}\text { Corrected mean } \\
\text { motion }\end{array}$ \\
\hline$t_{k}=t-t_{0 e}$ & $\begin{array}{l}\text { Time according to } \\
t_{0 e}\end{array}$ \\
\hline$M_{k}=M_{0}+n \cdot t_{k}$ & Mean anomaly \\
\hline$E_{k}=M_{k}+e \cdot \sin E_{k}$ & Eccentric anomaly \\
\hline$V_{K}=\tan ^{-1}\left(\frac{\sqrt{1-e^{2}} \cdot \sin E_{k}}{\cos E_{k}-e}\right)$ & True anomaly \\
\hline$V_{K}=\cos ^{-1}\left(\frac{e+\cos f_{n}}{1+e \cos f_{n}}\right)$ & True anomaly \\
\hline$U_{k}=\omega+V_{k}$ & $\begin{array}{l}\text { Argument } \\
\text { latitude }\end{array}$ \\
\hline$\delta U_{k}=C_{u c} \cdot \cos 2 U_{k}+C_{u s} \cdot \sin 2 U_{k}$ & $\begin{array}{l}\text { Argument of } \\
\text { latitude correction }\end{array}$ \\
\hline$\delta r_{k}=C_{r c} \cdot \cos 2 U_{k}+C_{r s} \sin 2 U_{k}$ & Radius correction \\
\hline$\delta i_{k}=C_{i c} \cdot \cos 2 U_{k}+C_{i s} \sin 2 U_{k}$ & $\begin{array}{l}\text { Inclination } \\
\text { correction }\end{array}$ \\
\hline$\Phi_{k}=U_{k}+\delta U_{k}$ & $\begin{array}{l}\text { Corrected argument } \\
\text { of latitude }\end{array}$ \\
\hline$r_{k}=a\left(1-e \cdot \cos E_{k}\right)+\delta r_{k}$ & Corrected radius \\
\hline$i_{k}=i_{o}+i . t_{k}+\delta i_{k}$ & $\begin{array}{l}\text { Corrected } \\
\text { inclination }\end{array}$ \\
\hline$\Omega_{k}=\Omega_{0}+\left(\dot{\Omega}-\dot{\Omega}_{e}\right) t_{k}-\dot{\Omega}_{e} t_{o e}$ & $\begin{array}{l}\text { Corrected longitude } \\
\text { of ascending node }\end{array}$ \\
\hline$X_{k}^{\prime}=r_{k} \cdot \cos \Phi_{k}$ & Position in the \\
\hline$Y_{k}^{\prime}=r_{k} \cdot \sin \Phi_{k}$ & orbi \\
\hline$X_{k}=X_{k}^{\prime} \cdot \cos \Omega_{k}-Y_{k}^{\prime} \cdot \sin \Omega_{k} \cdot \cos i_{k}$ & \\
\hline$Y_{k}=K_{k}^{\prime} \cdot \sin \Omega_{k}+Y_{k}^{\prime} \cdot \cos \Omega_{k} \cdot \cos i_{k}$ & geocentric satellite \\
\hline$Z_{k}=Y_{l}$ & \\
\hline
\end{tabular}

\section{RESULTS AND DISCUSSION}

For a comparison, the data obtained from a broadcast ephemeris file belonging to the selected time and all the coordinates in the ECEF system belonging to all the satellites were computed using the algorithm (see Table 5 ) and the results are given in Table 6 . Since broadcast ephemerides information belonging to satellites 19 and 28 did not exist for the time we studied, it was not included in the computations and comparisons.

Table 6 : Coordinates Computed from Broadcast Ephemeris Data

\begin{tabular}{|c|c|c|c|}
\hline \multirow[b]{3}{*}{ Sv } & \multicolumn{3}{|c|}{ BROADCAST EPHEMERIDES } \\
\hline & \multicolumn{3}{|c|}{$\begin{array}{c}\text { Date }: 22.09 .2015 \\
\text { Time : } 12 \quad 00 \quad 00.0 \text { (UTC) }\end{array}$} \\
\hline & $\mathbf{X}(\mathbf{m})$ & $\mathbf{Y}(\mathbf{m})$ & $\mathbf{Z}(\mathbf{m})$ \\
\hline $\mathrm{PG} 01 / 1$ & 13241509.1112 & -22684517.4902 & -3185779.0525 \\
\hline $\mathrm{PG} 02 / 2$ & -19062942.1707 & 8040568.1264 & -16021004.7228 \\
\hline $\mathrm{PG} 03 / 3$ & 10524879.3394 & -13434155.3029 & -20348279.9791 \\
\hline PG04 / 4 & 17487635.7595 & -18899496.8878 & 5580890.8762 \\
\hline PG05 / 5 & -25726352.4852 & 2709436.6092 & 6395764.0177 \\
\hline PG06 / 6 & -15102048.5066 & -4355823.4206 & -21409473.3875 \\
\hline PG07 / 7 & 1496332.7481 & -20904086.3120 & 16200889.5707 \\
\hline $\mathrm{PG} 08 / 8$ & 9613449.6557 & -13567567.5895 & 20756256.7077 \\
\hline $\mathrm{PG} 09 / 9$ & -6982217.0162 & -24840578.1163 & -6271115.2489 \\
\hline $\begin{array}{c}\text { PG10/ } \\
10\end{array}$ & 18020769.3446 & 3708296.6187 & 19272097.6589 \\
\hline $\begin{array}{c}\mathrm{PG} 11 / \\
11\end{array}$ & 10220819.5620 & -23855161.8523 & 4866821.5666 \\
\hline $\begin{array}{c}\text { PG12/ } \\
12 \\
\end{array}$ & -13429111.3494 & 11403133.8642 & -19910724.6289 \\
\hline $\begin{array}{c}\text { PG13/ } \\
13\end{array}$ & -14751728.3410 & 2703241.8466 & 21782990.8531 \\
\hline $\begin{array}{c}\text { PG14/ } \\
14\end{array}$ & 14467152.0676 & 20339524.5241 & -9046264.9330 \\
\hline $\begin{array}{c}\text { PG15/ } \\
15 \\
\end{array}$ & -9148103.2099 & 14508567.5799 & 20105616.2170 \\
\hline $\begin{array}{c}\text { PG16/ } \\
16 \\
\end{array}$ & 25972823.0282 & -282111.9732 & 6335651.6228 \\
\hline $\begin{array}{c}\text { PG17/ } \\
17 \\
\end{array}$ & -15209695.7659 & -19577725.9601 & -9512594.6810 \\
\hline $\begin{array}{c}\text { PG18/ } \\
18\end{array}$ & 9998746.5952 & 15467674.9555 & 19733909.5573 \\
\hline $\begin{array}{c}\text { PG20/ } \\
20\end{array}$ & -13952448.9143 & 13459192.4687 & 18035077.8103 \\
\hline $\begin{array}{c}\mathrm{PG} 21 / \\
21\end{array}$ & 3030146.4229 & 19664885.4718 & 18152398.9758 \\
\hline $\begin{array}{c}\text { PG22/ } \\
22\end{array}$ & 21902903.8949 & 10072173.1631 & 11577960.4784 \\
\hline $\begin{array}{c}\text { PG23/ } \\
23\end{array}$ & 4016930.7221 & -19851266.3489 & -16786537.5994 \\
\hline $\begin{array}{c}\text { PG24 / } \\
24 \\
\end{array}$ & -14556384.6682 & 22111328.9425 & -224097.5724 \\
\hline $\begin{array}{c}\text { PG25/ } \\
25 \\
\end{array}$ & 1034402.1893 & 14955831.4442 & -22033883.4358 \\
\hline $\begin{array}{c}\text { PG26/ } \\
26\end{array}$ & 25790651.8787 & 4884605.0664 & -4080593.8351 \\
\hline $\begin{array}{c}\text { PG27/ } \\
27\end{array}$ & 15971808.8445 & -394385.3141 & 21211096.3291 \\
\hline $\begin{array}{c}\text { PG29/ } \\
29 \\
\end{array}$ & 3925251.3408 & 24880784.6118 & -8473995.8229 \\
\hline $\begin{array}{c}\text { PG30/ } \\
30\end{array}$ & -7846485.3755 & -14067794.7995 & 21104373.4715 \\
\hline $\begin{array}{c}\text { PG31/ } \\
31 \\
\end{array}$ & 15397134.8865 & 3526605.8571 & -21275951.9003 \\
\hline $\begin{array}{c}\mathrm{PG} 32 / \\
32\end{array}$ & 21311233.5969 & -6694899.2395 & -13990519.1669 \\
\hline
\end{tabular}

Satellite coordinates existing in current IGS final ephemerides file are given in Table 7. 
Vol; 3; , Issue; 1, pp. 012-019, February, 2018,

Table 7 : Coordinates Taken from IGS Final Ephemerides File

\begin{tabular}{|c|c|c|c|}
\hline \multirow[b]{3}{*}{ SV } & \multicolumn{3}{|c|}{ IGS FINAL EPHEMERIDES (PG) } \\
\hline & \multicolumn{3}{|c|}{ 22.09.2015 - $1200 \quad 00.0$ (UTC) } \\
\hline & $\mathbf{X}(\mathbf{k m})$ & Y (km) & $\mathbf{Z}(\mathbf{k m})$ \\
\hline $\begin{array}{c}\text { PG01 / } \\
1\end{array}$ & 13241.510267 & -22684.518437 & -3185.778312 \\
\hline $\begin{array}{c}\mathrm{PG} 02 / \\
2\end{array}$ & -19062.942489 & 8040.569306 & -16021.003732 \\
\hline $\begin{array}{c}\text { PG03 / } \\
3 \\
\end{array}$ & 10524.880813 & -13434.156207 & -20348.279989 \\
\hline $\begin{array}{c}\text { PG04 / } \\
4 \\
\end{array}$ & 17487.636021 & -18899.497890 & 5580.890525 \\
\hline $\begin{array}{c}\text { PG05 / } \\
5\end{array}$ & -25726.352210 & 2709.437346 & 6395.764498 \\
\hline $\begin{array}{c}\text { PG06 / } \\
6\end{array}$ & -15102.049290 & -4355.823474 & -21409.474085 \\
\hline $\begin{array}{c}\text { PG07 / } \\
7\end{array}$ & 1496.332264 & -20904.086346 & 16200.889743 \\
\hline $\begin{array}{c}\text { PG08 / } \\
8\end{array}$ & 9613.449439 & -13567.568723 & 20756.257558 \\
\hline $\begin{array}{c}\text { PG09 / } \\
9\end{array}$ & -6982.216570 & -24840.579318 & -6271.115865 \\
\hline $\begin{array}{c}\text { PG10 / } \\
10\end{array}$ & 18020.769915 & 3708.298193 & 19272.098083 \\
\hline $\begin{array}{c}\text { PG11 / } \\
11\end{array}$ & 10220.820960 & -23855.162761 & 4866.822869 \\
\hline $\begin{array}{c}\text { PG12 / } \\
12\end{array}$ & -13429.111902 & 11403.134108 & -19910.723909 \\
\hline $\begin{array}{c}\text { PG13 / } \\
13\end{array}$ & -14751.729370 & 2703.244991 & 21782.991689 \\
\hline $\begin{array}{c}\text { PG14 / } \\
14 \\
\end{array}$ & 14467.151791 & 20339.526524 & -9046.265445 \\
\hline $\begin{array}{c}\text { PG15 / } \\
15 \\
\end{array}$ & -9148.104842 & 14508.566576 & 20105.615974 \\
\hline $\begin{array}{c}\text { PG16/ } \\
16\end{array}$ & 25972.824222 & -282.113556 & 6335.654492 \\
\hline $\begin{array}{c}\text { PG17 / } \\
17 \\
\end{array}$ & -15209.694542 & -19577.726766 & -9512.594024 \\
\hline $\begin{array}{c}\text { PG18 / } \\
18 \\
\end{array}$ & 9998.748167 & 15467.676057 & 19733.910223 \\
\hline $\begin{array}{c}\mathrm{PG} 20 / \\
20\end{array}$ & -13952.450041 & 13459.192872 & 18035.078960 \\
\hline $\begin{array}{c}\text { PG21 / } \\
21 \\
\end{array}$ & 3030.148062 & 19664.885926 & 18152.400788 \\
\hline $\begin{array}{c}\text { PG22 / } \\
22\end{array}$ & 21902.903891 & 10072.173885 & 11577.959609 \\
\hline $\begin{array}{c}\text { PG23 / } \\
23 \\
\end{array}$ & 4016.930809 & -19851.266599 & -16786.537380 \\
\hline $\begin{array}{c}\text { PG24 / } \\
24\end{array}$ & -14556.385671 & 22111.329422 & -224.097685 \\
\hline $\begin{array}{c}\text { PG25 / } \\
25\end{array}$ & 1034.402967 & 14955.832070 & -22033.884220 \\
\hline $\begin{array}{c}\text { PG26/ } \\
26\end{array}$ & 25790.653160 & 4884.605494 & -4080.593578 \\
\hline $\begin{array}{c}\text { PG27 / } \\
27\end{array}$ & 15971.809573 & -394.384769 & 21211.097228 \\
\hline $\begin{array}{c}\text { PG29 / } \\
29\end{array}$ & 3925.250834 & 24880.784699 & -8473.995905 \\
\hline $\begin{array}{c}\text { PG30 / } \\
30\end{array}$ & -7846.486155 & -14067.795260 & 21104.374161 \\
\hline $\begin{array}{c}\text { PG31 / } \\
31\end{array}$ & 15397.134642 & 3526.606158 & -21275.951929 \\
\hline $\begin{array}{c}\text { PG32 / } \\
32\end{array}$ & 21311.233542 & -6694.900451 & -13990.520418 \\
\hline
\end{tabular}

When the coordinates obtained from two different types of ephemerides information were compared, the differences in Table 8 were reached and they were shown in Figure 5 .
Table 8 : Differences in Coordinates Obtained from Broadcast Ephemeris and IGS Final Ephemeris Data

\begin{tabular}{|c|c|c|c|}
\hline & \multicolumn{3}{|c|}{ DIFFERENCES } \\
\hline SV & $\boldsymbol{\Delta X}(\mathbf{m})$ & $\boldsymbol{\Delta} \mathbf{( m )}$ & $\boldsymbol{\Delta Z} \mathbf{( m )}$ \\
\hline PG01 / 1 & 1.1558 & -0.9468 & 0.7405 \\
\hline PG02 / 2 & -0.3183 & 1.1796 & 0.9908 \\
\hline PG03 / 3 & 1.4736 & -0.9041 & -0.0099 \\
\hline PG04 / 4 & 0.2615 & -1.0022 & -0.3512 \\
\hline PG05 / 5 & 0.2752 & 0.7368 & 0.4803 \\
\hline PG06 / 6 & -0.7834 & -0.0534 & -0.6975 \\
\hline PG07 / 7 & -0.4841 & -0.0340 & 0.1723 \\
\hline PG08 / 8 & -0.2167 & -1.1335 & 0.8503 \\
\hline PG09 / 9 & 0.4462 & -1.2017 & -0.6161 \\
\hline PG10 / 10 & 0.5704 & 1.5743 & 0.4241 \\
\hline PG11 / 11 & 1.3980 & -0.9087 & 1.3024 \\
\hline PG12 / 12 & -0.5526 & 0.2438 & 0.7199 \\
\hline PG13 / 13 & -1.0290 & 3.1444 & 0.8359 \\
\hline PG14 / 14 & -0.2766 & 1.9999 & -0.5120 \\
\hline PG15 / 15 & -1.6321 & -1.0039 & -0.2430 \\
\hline PG16 / 16 & 1.1938 & -1.5828 & 2.8692 \\
\hline PG17 / 17 & 1.2239 & -0.8059 & 0.6570 \\
\hline PG18 / 18 & 1.5718 & 1.1015 & 0.6657 \\
\hline PG20 / 20 & -1.1267 & 0.4033 & 1.1497 \\
\hline PG21 / 21 & 1.6391 & 0.4542 & 1.8122 \\
\hline PG22 / 22 & -0.0039 & 0.7219 & -0.8694 \\
\hline PG23 / 23 & 0.0869 & -0.2502 & 0.2194 \\
\hline PG24 / 24 & -1.0028 & 0.4795 & -0.1126 \\
\hline PG25 / 25 & 0.7777 & 0.6258 & -0.7842 \\
\hline PG26 / 26 & 1.2813 & 0.4276 & 0.2571 \\
\hline PG27 / 27 & 0.7285 & 0.5451 & 0.8989 \\
\hline PG29 / 29 & -0.5068 & 0.0872 & -0.0821 \\
\hline PG30 / 30 & -0.7795 & -0.4605 & 0.6895 \\
\hline PG31 / 31 & -0.2445 & 0.3009 & -0.0287 \\
\hline PG32 / 32 & -0.0549 & -1.2115 & -1.2511 \\
\hline Maximum & 1.6391 & 3.1444 & 2.8692 \\
\hline Minimum & -1.6321 & -1.5828 & -1.2511 \\
\hline Mean & 0.1691 & 0.0842 & 0.3392 \\
\hline Standard & 0.9180 & 1.0769 & 0.8617 \\
\hline Deviations & & & \\
\hline
\end{tabular}

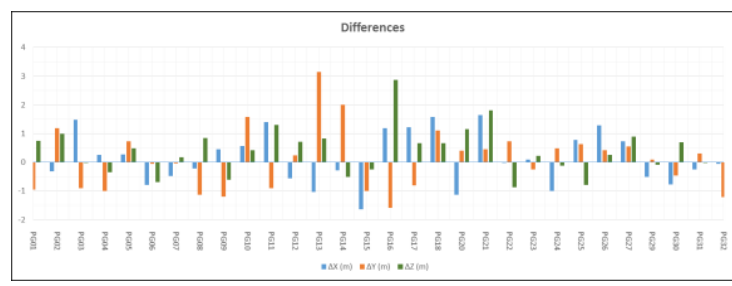

Figure 5 : Differences of ECEF satellite coordinates computed from broadcast ephemerides from IGS final ephemerides

When Table 8 is examined, it is seen that the differences between the coordinate components of broadcast and final ephemerides vary between $-1,6321$ 
$\mathrm{m}$ and $+1,6391 \mathrm{~m}$ on the $\mathrm{X}$-axis, between $-1,5828 \mathrm{~m}$ and $+3,1444 \mathrm{~m}$ on the $\mathrm{Y}$-axis and between $-1,2511 \mathrm{~m}$ and $2,8692 \mathrm{~m}$ on the $\mathrm{Z}$-axis. When the means of these differences are taken into consideration, it appears that $\mathrm{X}$ coordinate component has an average difference of $\sim 17$ $\mathrm{cm}, \mathrm{Y}$ coordinate component $\sim 8 \mathrm{~cm}$, and $\mathrm{Z}$ coordinate component $\sim 34 \mathrm{~cm}$. On the other hand, when the standard deviations of computations are taken into consideration it can be seen from Table 8 that the mean standard deviation values are $0,9180 \mathrm{~m}, 1,0769 \mathrm{~m}, 0,8617 \mathrm{~m}$ for $\mathrm{X}, \mathrm{Y}$ and $\mathrm{Z}$ components, respectively.

Numerous studies have been conducted from past to present with regard to orbital accuracy and its improvement. FGCC (Federal Geodetic Control Committee) stated in a guidebook entitled Geometric Geodetic Accuracy Standards and Specifications for Using GPS Relative Positioning Techniques published in 1989 that there were small differences between final ephemerides and broadcast ephemerides and that this accuracy was enough to meet the needs of most engineering projects. According to Rui-xi et al. (2014), accuracy of broadcast ephemerides was found to be around $\pm 1 \mathrm{~m}$ for each of the $\mathrm{X}, \mathrm{Y}$ and $\mathrm{Z}$ coordinates. Likewise, it was stated in Grzegorz et al. (2015) and in IGS (2017) that satellite coordinates could be obtained with an accuracy of $\pm 1 \mathrm{~m}$ through broadcast ephemerides.

\section{CONCLUSION}

Reliable, consistent positioning accuracy has always driven new product development in the survey and mapping sector of the GNSS market (Cameron 2015). It should not be forgotten that accuracy of the coordinates calculated from broadcast and final ephemerides data is influenced by gravity field information and tropospheric and ionospheric factors. ITRF system, which uses GRS80 reference ellipsoid, is used in productions made from final ephemerides whereas WGS84 system, which again uses GRS80 reference ellipsoid in calculations made from broadcast ephemerides data, but it is pointed out that there is not much difference between WGS84 and ITRF systems in practical applications (Stanaway 2007).

When all the effects are taken into consideration, the desired precision in the study to be conducted needs to be determined properly before deciding on the ephemerides information to be used. Use of IGS final ephemerides data in specific studies such as establishment of first degree Networks and deformation measurements which require extremely high precision may ensure that results have even higher levels of accuracy. For example, it would be appropriate to use IGS final ephemerides data to improve accuracies of ITRF coordinates of local/regional geodetic studies. On the other hand, release period of IGS products ranging from about 1 day to 2 weeks is a major disadvantage for high precision GNSS processing. Therefore, the fact that broadcast ephemerides data can be accessed at any time and that it yields results that are not much different from those obtained from final ephemerides data render broadcast ephemerides a more easily applied alternative in many practical applications.

\section{REFERENCES}

Bidikar, B., Rao, G.S., Ganesh, L., Santosh Kumar, MNVS., 2014. Satellite Clock Error and Orbital Solution Error Estimation for Precise Navigation Applications. Positioning, 2014, 5, 22-26

Cameron, A., 2015, "More, More, More. Accuracy, Accuracy, Accuracy." GPS World, September 2, 2015, [Access Date; 14.08.2017]

Federal Geodetic Control Committee (FGCC), 1989. Geometric Geodetic Accuracy Standards and Specifications For Using GPS Relative Positioning Techniques. Federal Geodetic Control Committee, Version 5.0, p.48

Grzegorz, N., Mariusz, F., Zofia, B., 2015. Comparison of GPS Precise Ephemerides Interpolation Methods. 15th International Multidisciplinary Scientific GeoConferences SGEM2015, Section Geodesy and Mine Surveying, pp 161-170, DOI: 10.5593/SGEM2015/B22/S9.020

Hilla, S., 2010. The Extended Standard Product 3 Orbit Format (SP3-c). National Geodetic Survey National Ocean Service, NOAA, Silver Spring, MD 20910-6233, USA,https://igscb.jpl.nasa.gov/igscb/data/format/sp3c.tx t, [Access Date; 24.07.2017].

Hofmann-Wellenhof, B., Lichtenegger, H. and Collins, J., 1994. GPS - Theory and Practice. 3rd Edition, SpringerVerlag Wein New York.

ICD, 1993. Interface Control Document - NAVSTAR GPS Space Segment/Navigation User Interfaces (ICDGPS-200C). ARINC Research Corporation, Revision IRN-200C-004, 12 April 2000.

IGS, 2017. International GNSS Service. http://www.igs.org/, [Access Date; 14.08.2017]

Kahveci, M., Yildiz, F., 2001. Global Positioning System Theory-Applications. Nobel Publisher, p.184, Ankara, (in Turkish).

Parkinson, B., Spilker, J. 1996. Global Positioning System: Theory and Applications. American Institute of Aeronautics and Astronautics, Volume U, p.643, Washington D.C.

Remondi, BW. 1991. NGS Second Generation ASCII and Binary Orbit Formats and Associated Interpolated Studies. Proceedings of the Twentieth General Assembly, International Union of Geodesy and Geophysics, Vienna, Austria, August 11-24, 1991, 28 pp.

Rui-xi, J., Xiao-yu, L., Chang-feng, X and Dong-yang, J., 2014. Broadcast Ephemerides Accuracy Analysis for GPS Based on Precise Ephemerides, Applied Mechanics and Materials Vols 602-605 (2014), pp 3667-3670.

Seeber, G., 1993. Satellite Geodesy: Foundations, Methods, and Applications. W. De Gruyter, p.531, Berlin, New York. 
Stanaway, R., 2007, GDA94, ITRF \& WGS84 What's the difference? Working with Dynamic Datums, SSC 2007,

Hobart, Tasmania, Australia

Tusat, E., Turgut. B., 2003. GPS Ephemerides Effects of Relative Positioning on the Coordinates and Baseline Components. Selcuk University Journal of Engineering, Science and Technology, Vol.19, No.1, pp:65-72, Konya, (in Turkish).

Warren, DLM., 2002. Broadcast vs Precise GPS Ephemerides: A Historical Perspective. Master Thesis, Department Of The Air Force Air University Air Force Institute Of Technology, p.169, Ohio, USA.

Yoon, S., 2015. Precise GPS Orbit Determination at National Geodetic Survey: How and Why. 55th Civil GPS Service Interface Committee (CGSIC) Meeting, At the Institute of Navigation GNSS+ 2015 Conference Tampa Convention Center 14-15 September 2015, Tampa, Florida.

Copyright (C) International Journal of Engineering and Geosciences (IJEG). All rights reserved, including the making of copies unless permission is obtained from the copyright proprietors. 